\title{
Entwöhnungsbehandlung Alkoholabhängiger und andere Formen der Postakutbehandlung
}

\author{
Heribert Fleischmann
}

Die Entwöhnungsbehandlung ist eine erfolgreiche Reha-Maßnahme Alkoholabhängiger mit dem Ziel der Abstinenz. Sie arbeitet mit interdisziplinären und multimodalen Interventionen und umfasst so alle Dimensionen des biopsychosozialen Krankheitsmodells.

\section{Abkürzungen \\ CMA chronisch mehrfach Abhängige \\ DGSS Deutsche Gesellschaft für Suchtforschung und Suchttherapie \\ DRV Deutsche Rentenversicherung \\ EWB Entwöhnungsbehandlung \\ ICF Internationale Klassifikation der \\ Funktionsfähigkeit, Behinderung und Gesundheit \\ KVT kognitive Verhaltenstherapie \\ KTL Klassifikation therapeutischer Leistungen \\ LoE Level of Evidence \\ MI motivationale Interventionsformen \\ PKV private Krankenversicherung \\ PSB psychosoziale Behandlungs- und Beratungsstellen \\ SGB Sozialgesetzbuch}

\section{Verortung der Entwöhnungs- behandlung im Versorgungs- system}

Riskant Alkohol konsumierende Menschen entwickeln Störungen, mit denen sie aus unterschiedlichen Anlässen in Kontakt mit den Hilfesystemen kommen (Abb. 1). Für suchtkranke Menschen steht in Deutschland ein differenziertes Beratungs- und Behandlungssystem zur Verfügung. Ziel ist, die Beratungs- und Behandlungsangebote indikationsbezogen, bedarfsgerecht, patientenorientiert und mit hoher Qualität zu erbringen. Krankheitsbeschwerden werden nach dem biomedizinischen System der ICD-10 klassifiziert. Bei Behandlungsbedarf werden Patienten einer ambulanten oder (teil-)stationären kurativen Behandlung (Akutbehandlung) zugeführt (Abb.2). Die Finanzierung ist im Sozialgesetzbuch (SGB) V geregelt [1, 2].

\section{Die häufigste Anschlussbehandlung (Postakutbe- handlung) ist die medizinische Rehabilitation des Abhängigkeitssyndroms (Abb. 2).}

An die Postakutbehandlung können sich wiederum Maßnahmen unterschiedlichster Art anschließen (s.u.). Für die Finanzierung der Entwöhnungsbehandlung (EWB) ist vorrangig die Deutsche Rentenversicherung (DRV), geregelt im SGB VI, zuständig. Die Klassifikation der Funktionsstörungen erfolgt im biopsychosozialen System der ICF (Internationale Klassifikation der Funktionsfähigkeit, Behinderung und Gesundheit) (Abb.3) $[1,3]$. Die Überbrückung der Schnittstelle zwischen Krankenkassen (Akutbehandlung, SGB V) und Rentenversicherungsträgern (medizinische Rehabilitation, SGB VI) regelt die Vereinbarung Abhängigkeitskranker [2]. Aufgrund der Vielfalt der Störungsmuster, Behandlungskonzepte, der institutionellen Gliederung der Behandlungsangebote und Leistungserbringer, der 


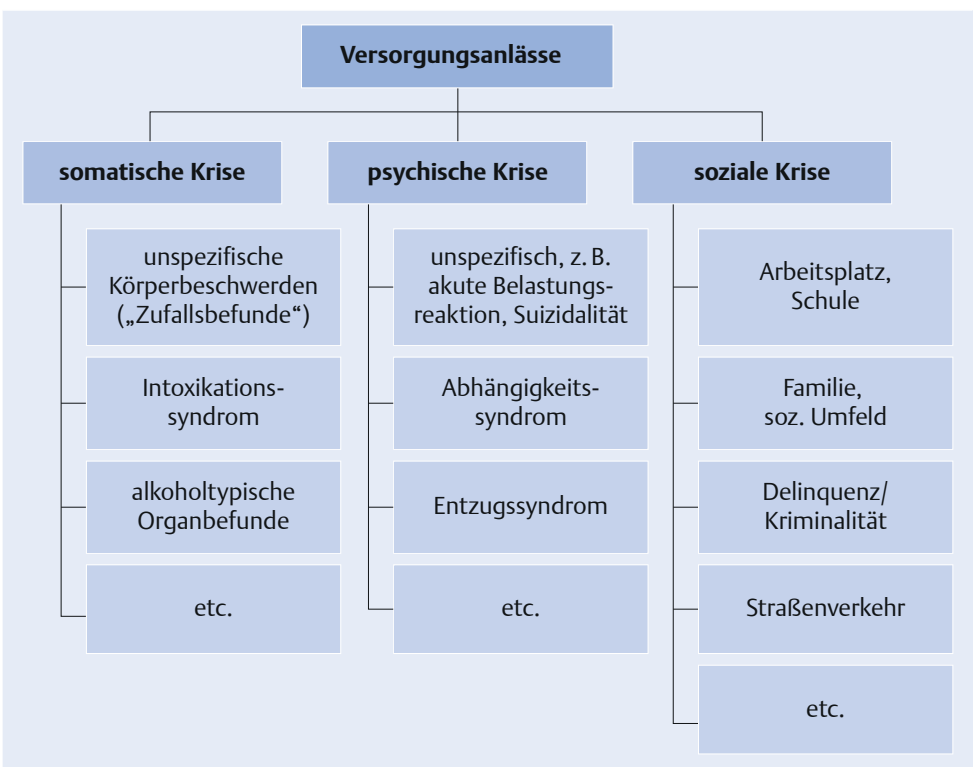

Abb. 1 Versorgungsanlässe und Zugangswege [7].

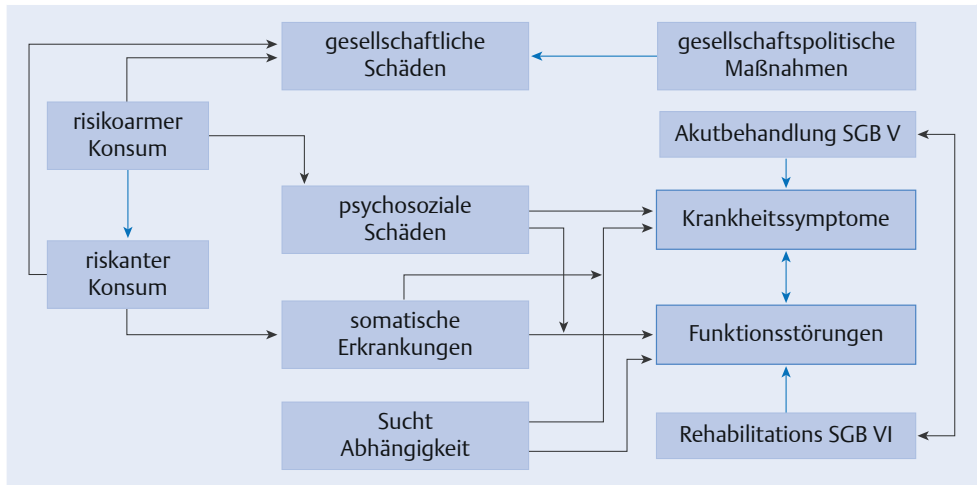

Abb. 2 Verortung der Rehabilitation im medizinischen Hilfssystem.

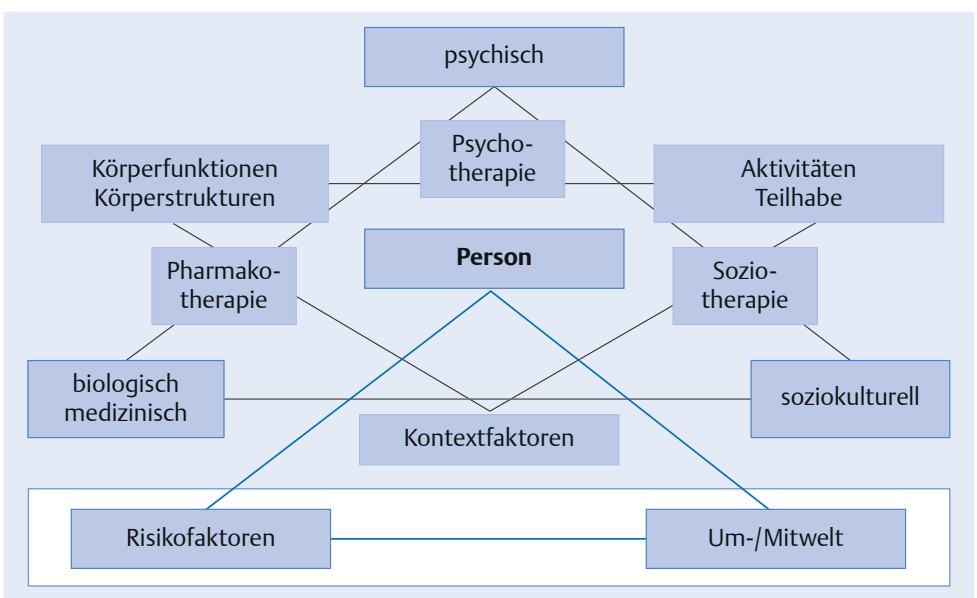

Abb. 3 Das biopsychosoziale System.
Indikation kurativer vs. rehabilitativer Behandlung und der Zuständigkeit der Kostenträger entstehen Schnittstellen, die ein differenziertes Überleitungsmanagement erfordern (Abb.4). Das stationäre Behandlungsangebot wird jährlich von ca. 46000 Rehabilitanden, davon $70 \%$ wegen Alkohol, die ambulante EWB von 7000 - 8000 Rehabilitanden in Anspruch genommen $[4,5]$.

Sozialmedizinisch werden kurative (Akutbehandlung) und rehabilitative Behandlung (Postakutbehandlung) differenziert.

\section{Voraussetzungen für die Indikationsstellung einer EWB}

Nicht alle Suchtkranken haben den aufwendigen Hilfebedarf einer EWB oder verfügen über die nötigen Voraussetzungen für deren erfolgreiche Durchführung. Die Indikation ist deshalb sorgfältig zu stellen. Bei der Planung der Leistungserbringung ist ggf. nach Geschlecht, Alter, Migrationshintergrund, Komorbidität (z.B. Beigebrauch illegaler Suchtstoffe, Glücksspiel u.a.) zu differenzieren (Tab. 1). Da die geburtenstarken Jahrgänge (1950 - 1970) im Zeitraum des seit 1965 anhaltenden Hochdosiskonsums (Pro-Kopf-Verbrauch reiner Alkohol ca. 11 Liter) sozialisiert sind, ist in Zukunft verstärkt mit Alkoholkranken über 65 Jahren zu rechnen [4]. Für die Überbrückung der Schnittstelle „kurative/rehabilitative Behandlung“ sind sozialmedizinische und versicherungsrechtliche Voraussetzungen zu klären (Überleitung) [5]. Dafür ist eine multiprofessionelle Kooperation zwischen ärztlichen und sozialpädagogischen Fachkräften erforderlich.

\section{Versicherungsrechtliche Voraussetzungen}

Leistungsberechtigt ist, wer Mindestversicherungszeiten aufweist und definierte Beitragszahlungen aus versicherungspflichtiger Arbeit geleistet hat. Die zuständige Krankenkasse kommt nur subsidiär zum Tragen. Besondere Aufmerksamkeit ist bei Mitgliedern privater Krankenversicherungen (PKV) geboten, da diese oft keinen Anspruch an einen Rentenversicherungsträger haben und für Reha-Leistungen Ausschlussklauseln der PKV gelten. Für die Leistungszuweisung ist das Antragsund Bewilligungsverfahren der DRV zu beachten [2]. Neben dem ärztlichen Gutachten zum Reha-Antrag mit Darlegung der funktionellen Beeinträchtigungen (Abb. 3) ist ein Sozialbericht erforderlich, aus dem detailliert 


\section{Tabelle 1}

Komorbiditätsspektrum von Patienten in stationärer Behandlung.

\begin{tabular}{|c|c|}
\hline Komorbidität & Häufigkeit \\
\hline \multicolumn{2}{|l|}{ mit somatischen Erkrankungen [29] } \\
\hline hepatologische Begleiterkrankungen & $47 \%$ \\
\hline Polyneuropathie & $16 \%$ \\
\hline \multicolumn{2}{|l|}{ mit psychischen Erkrankungen [9] } \\
\hline psychotische Störung & $8 \%$ \\
\hline Suizidrate & bis $4 \%$ \\
\hline $\begin{array}{l}\text { affektive Erkrankungen (bipolar und/oder } \\
\text { Depression) }\end{array}$ & $13,4 \%$ \\
\hline $\begin{array}{l}\text { Depression bei Männern (Life-Time-Diagno- } \\
\text { sen) }\end{array}$ & $24,3 \%$ \\
\hline Depression bei Frauen (Life-Time-Diagnosen) & $48,5 \%$ \\
\hline Angststörungen & $31-42 \%$ \\
\hline PTBS (Männer und Frauen) & $15-25 \%$ \\
\hline PTBS (Frauen) & bis $48 \%$ \\
\hline Persönlichkeitsstörung (F6 der ICD-10) & $?$ \\
\hline Demenz (F0 der ICD-10) & $?$ \\
\hline Intelligenzminderungen (F7 der ICD-10) & ? \\
\hline \multicolumn{2}{|l|}{ mit anderen Abhängigkeiten [3] } \\
\hline Tabak (F17) & $70,0 \%$ \\
\hline Cannabis (F12) & $10,6 \%$ \\
\hline Stimulanzien (F15) & $6,7 \%$ \\
\hline $\begin{array}{l}\text { Sedativa, Hypnotika, inkl. Benzodiazepine } \\
\text { (F13) }\end{array}$ & $4,2 \%$ \\
\hline Kokain (F14) & $3,5 \%$ \\
\hline Opioide (F11) & $3,3 \%$ \\
\hline Halluzinogene (F16) & $1,4 \%$ \\
\hline nicht stoffgebundene Sucht (z. B. Glücksspiel) & ? \\
\hline
\end{tabular}

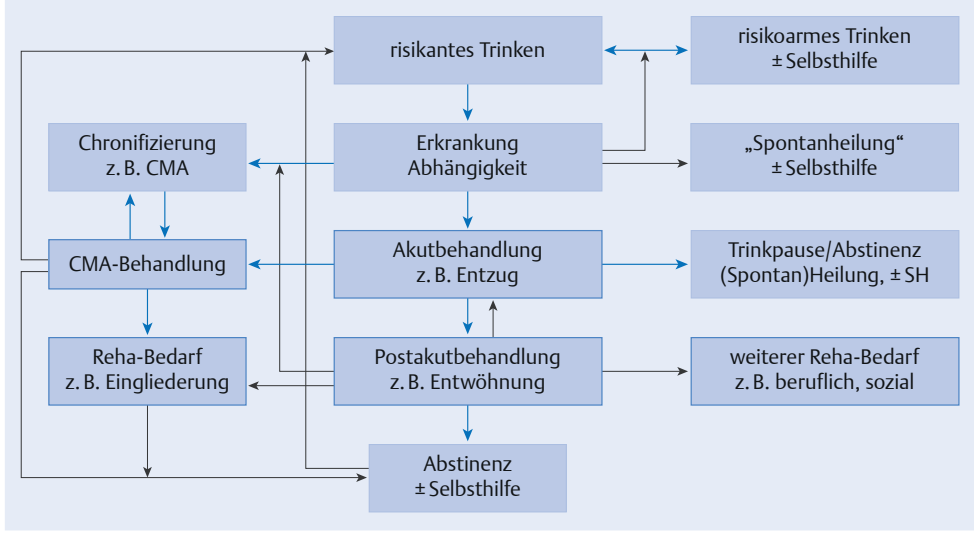

Abb.4 Versorgungswege des medizinischen Hilfesystems.

der bisherige Krankheitsverlauf und insbesondere die psychosozialen Auswirkungen der Erkrankung zu entnehmen sind [5]. Der Rententräger entscheidet nach pflichtgemäßem Ermessen über Art, Ort und Dauer der EWB.

Im Unterschied zur Akutbehandlung ist der Zugang zur EBW nicht über eine ärztliche Einweisung, sondern nur elektiv durch den Rententräger möglich.

\section{Gesundheitliche Voraussetzungen}

Um eine EWB in Anspruch nehmen zu können, müssen folgende gesundheitliche und motivationale Voraussetzungen erfüllt sein:

- Reha-Bedürftigkeit: Es muss eine Alkoholabhängigkeit mit daraus resultierenden Funktionsstörungen und Risiken vorliegen.

- Reha-Fähigkeit: Der Antragsteller sollte für die Behandlung bereit und motiviert sowie ausreichend psychisch und körperlich belastbar sein.

- günstige Prognose: Das Rehabilitationsziel „Abstinenz" muss mit Wahrscheinlichkeit erreichbar sein.

- Maßnahmen der Beratung und Motivierung müssen im Vorfeld erfolgt sein.

- Der körperliche Entzug muss abgeschlossen sein, d.h. der Patient befindet sich im Zustand einer „Trinkpause" und ist bereit, diese während der Behandlung einzuhalten [1]. Im Vorfeld einer EWB ist deshalb in der Regel eine Akutbehandlung (Entzug/Entgiftung) erforderlich (Abb.4) [2].

- Der Abhängigkeitskranke muss bereit sein, eine ggf. erforderliche Nachsorge in Anspruch zu nehmen. 


\section{Qualitative Voraussetzungen der Leistungserbringer}

Eine EWB kann nur in einer ambulanten oder stationären Einrichtung durchgeführt werden, deren Qualität auf einem internen Qualitätsmanagement beruht $[2,5$, 6]. Struktur- und Prozessqualität müssen nach einem unabhängigen, von der DRV anerkannten Zertifizierungsverfahren geprüft sein. Ein Therapieprogramm entsprechend der Prozessleitlinie (Reha-TherapieStandards) muss schriftlich vorliegen [5, 6]. Die therapeutischen Leistungen müssen in der KTL (Klassifikation therapeutischer Leistungen) dokumentiert werden. Die Qualität des Reha-Verlaufs wird mit dem PeerReview-Verfahren (einheitlicher Reha-Entlassungsbericht, individuelle Therapieplanung) überprüft. Der Erfolg der Rehabilitation wird aus Patientensicht in regelmäßigen Befragungen ermittelt.

Für die spezifisch suchttherapeutischen Interventionen müssen bestimmte Berufsgruppen mit entsprechenden Aus- und Weiterbildungen eingesetzt werden. Dazu gehören:

- Fachärzte

- approbierte psychologische Psychotherapeuten oder Diplom-Psychologen mit suchttherapeutischen Weiterbildungen - entsprechend den Empfehlungen der Bundes- und Landesärztekammern oder dem Psychotherapeutengesetz

- Diplom-Sozialarbeiter/Diplom-Sozialpädagogen mit tätigkeitsfeldspezifischen Weiterbildungsgängen, die auf die Indikation Sucht ausgerichtet sind $[2,5]$.

Die nötigen Qualifikationen liegen für die ambulante Behandlung in der Regel in den Psychosozialen Behandlungs- und Beratungsstellen (PSB) vor. Für die stationäre und/oder ganztags ambulante Rehabilitation ist dies in den Suchtfachkliniken oder Tageskliniken, vereinzelt auch in der Suchtpsychiatrie gewährleistet.

Die von der DRV beauftragte Reha-Klinik teilt dem Rehabilitanden einen Aufnahmetermin mit. Das vorgesehene Rehabilitationsprogramm muss aus medizinischer Sicht für die Erreichung der individuellen Rehabilitationsziele geeignet sein und ggf. besondere Bedingungen wie Alter, Geschlecht, Migrationshintergrund und Komorbidität berücksichtigen.

Eine rehabilitative Behandlung wird auf Antrag vom Rentenversicherungsträger, subsidiär der zuständigen Krankenkasse oder dem Sozialhilfeträger nach pflichtgemäßem Ermessen bewilligt.

\section{Überleitungswege und Setting- Indikatoren}

Die EWB wird ambulant, ganztags ambulant (teilstationär) und stationär durchgeführt. Bei der Zuweisung zum Setting sind folgende Kriterien zu berücksichtigen: Ausmaß der biopsychosozialen Störungen (Abb.3), Dauer und Intensität der Abhängigkeitserkrankung, Beschaffenheit des sozialen Umfelds des Rehabilitanden (dysfunktional oder stabilisierend), Status der beruflichen Integration des Abhängigkeitskranken (vorhandener Arbeitsplatz oder Erwerbslosigkeit), stabile Wohnsituation, Fähigkeit zur aktiven Mitarbeit, zur regelmäßigen Teilnahme und zur Einhaltung des Therapieplans und Fähigkeit zur Einhaltung der Konsumpause $[2,5]$.

Aufgrund der unterschiedlichen Eingangs- bzw. Zuweisungsvoraussetzungen gibt es jedoch keine Empfehlungen, welches Setting grundsätzlich wirksamer ist.

\footnotetext{
Die Übereinstimmung zwischen Therapeut und Patient zur Auswahl des Settings verbessert das Therapieergebnis [7].
}

In die stationäre EWB werden die Rehabilitanden überwiegend aus den Beratungsstellen zugewiesen, in die ambulante Rehabilitation kommen sie aus eigenem Antrieb (Tab. 2).

\section{Grundsätze der rehabilitativen Behandlung}

Eine EWB ist ein komplexes therapeutisches Interventionspaket, das somatisch-biologische, psycho- und sozialtherapeutische Komponenten umfasst (Abb.3). Dieses steht als individuelles Programm dem Rehabilitanden zur Verfügung. Die Therapieplanung sollte grundsätzlich auf einem komplexen Interventionsansatz beruhen, auch wenn die Evidenz aufgrund methodologischer Probleme unvollständig, widersprüchlich oder nur schwach ausgeprägt ist [7].

\section{Die ICF als Grundlage der Rehabilitation}

Für die Akutbehandlung ist das biomedizinische Modell der ICD-10 die Behandlungsgrundlage, für die Rehabilitation die ICF [3, 8]. Die ICD-10 klassifiziert objekti- 
vierte Krankheitssymptome, z.B. Entzugsbeschwerden oder Symptome der Abhängigkeit wie Kontrollverlusterleben und Toleranzsteigerung, die ICF den Zustand der funktionalen Gesundheit eines Alkoholkranken als Ergebnis der Wechselwirkung zwischen Gesundheitsproblem und Kontextfaktoren wie Arbeit, Familie oder Freizeit.

Jede Beeinträchtigung der funktionalen Gesundheit wird im ICF „Behinderung“ genannt.

Behinderung ist nach ICF keine Eigenschaft einer Person, sondern wird in Relation zum Lebensumfeld eines Menschen beurteilt.

Nach diesem funktionalen Konzept von Gesundheit (Abb.3) gilt ein Alkoholkranker als gesund, wenn vor seinem Lebenshintergrund (Konzept der Kontextfaktoren)

- die körperlichen Funktionen (einschließlich des geistigen und seelischen Bereichs) und die Körperstrukturen allgemein anerkannten (statistischen) Normen entsprechen (Konzept der Körperfunktionen und -strukturen).

- er all das tut oder tun kann, was von einem Menschen ohne Gesundheitsprobleme (ICD-10) erwartet wird (Konzept der Aktivitäten).

- er sein Dasein in allen Lebensbereichen, die ihm wichtig sind, in der Weise und in dem Umfang entfalten kann, wie es von einem Menschen ohne Beeinträchtigungen der Körperfunktionen, Körperstrukturen oder der Aktivitäten erwartet wird (Konzept der Teilhabe an Lebensbereichen) $[1-3,5,9]$.

Die ICD-10 klassifiziert Krankheiten, die ICF Funktionsstörungen.

\section{Differenzierung der Behandlungsziele}

Arbeits- und Funktionsfähigkeit. Eine Rehabilitation wird nach der Eingangsdiagnostik auf der Basis der individuellen Erarbeitung der Rehabilitationsziele auf der Basis der ICF durchgeführt. Die Einbeziehung des Patienten bei der Entscheidungsfindung ist eine Grundbedingung des Erfolgs (partizipative Entscheidungsfindung).

Sozialmedizinisch ist im Unterschied zur Akutbehandlung (Wiederherstellung der Arbeitsfähigkeit) in der medizinischen Rehabilitation der Erhalt, die Verbesserung oder die Wiederherstellung der Funktions- und Leistungsfähigkeit sowie die Förderung der Teilhabe

\section{Tabelle 2}

Vermittlungswege (Jahresstatistik 2012 [30]).

\begin{tabular}{|c|c|c|}
\hline \multirow[b]{2}{*}{ Vermittlung durch } & \multicolumn{2}{|c|}{ Entwöhnungsbehandlung } \\
\hline & ambulant & stationär \\
\hline keine/Selbstzahler & $37,0 \%$ & $2,0 \%$ \\
\hline Familie & $7,7 \%$ & $2,0 \%$ \\
\hline Arbeitgeber, Betrieb, Schule & $3,0 \%$ & $1,8 \%$ \\
\hline ärztliche/psychotherapeutische Praxis & $5,3 \%$ & $1,6 \%$ \\
\hline Suchtberatungs-|-behandlungsstelle & $2,5 \%$ & $57,6 \%$ \\
\hline Institutsambulanz & $0,4 \%$ & $0,9 \%$ \\
\hline Krankenhaus(abteilung) & $12,3 \%$ & $21,0 \%$ \\
\hline stationäre Rehabilitationseinrichtungen & $6,6 \%$ & $5,3 \%$ \\
\hline ARGE/Jobcenter & $4,2 \%$ & $0,2 \%$ \\
\hline Justizbehörde/Bewährungshilfe & $5,3 \%$ & $2,9 \%$ \\
\hline Kostenträger/Leistungsträger & $2,3 \%$ & $0,6 \%$ \\
\hline
\end{tabular}

am Arbeitsleben und in der Gesellschaft das vorrangige Behandlungsziel. Angestrebt wird eine mehr als 6-stündige Belastbarkeit pro Arbeitstag.

Abstinenz. Das berufliche Teilhabeziel ist am ehesten zu erreichen, wenn der Rehabilitand zukünftig abstinent lebt, weshalb diese Zielsetzung von der DRV ausdrücklich gefordert wird ( $\$ 2$ der Vereinbarung „Abhängigkeitsmerkmale“ [5]).

Wenn der Rehabilitand trotz Aufklärung den Konsum fortsetzen oder lediglich eine mehr oder weniger lange zeitlich begrenzte Trinkpause einhalten will, kann das im therapeutischen Alltag paradoxerweise bedeuten, mit ihm zunächst Strategien zu erarbeiten, wie er den Konsum deutlich reduzieren oder ggf. „kontrollieren“ kann. Zu differenzieren sind deshalb folgende konsumbezogene Ziele:

- Trinkpausen

- Abstinenz

- Trinkmengenreduktion

- „kontrolliertes Trinken“

Konsumreduktion kann in bestimmten Fällen ein

Zwischenziel auf dem Weg zur Abstinenz sein. 
Abstinenz bedeutet die Beendigung jeglichen Alkoholkonsums aus eigenem Entschluss und Überzeugung aufgrund einer Einstellungs-und Haltungsänderung. Die intendierte Zeitdauer kann variabel sein und hängt von individuellen Konstellationen und weiteren Lebensereignissen ab. Von Rückfall sollte man nur dann sprechen, wenn eine intendierte Abstinenz und nicht eine Konsumpause beendet wird.

\section{Tipp für die Praxis}

Die Beendigung eines Abstinenzvorsatzes wird im Unterschied zur Wiederaufnahme des Trinkens nach einer Konsumpause in der Regel als Scheitern erlebt Im therapeutischen Prozess ist der Rehabilitand darauf vorzubereiten, um ihn zu befähigen, einen Rückfall mit und ohne therapeutische Hilfe schnell beenden zu können.

Konsumpausen. Trinkmengenreduktion oder „kontrolliertes Trinken“ können im Rahmen der Umorientierung ein Zwischenschritt zur Abstinenz sein [7, 10]. Gesundheitliche bzw. psychosoziale Probleme können darüber hinaus Konsumpausen unabdingbar nötig machen. Dazu gehören die Einnahme bestimmter Medikamente, ein Organversagen (Leber, Pankreas, Karzinome), die soziale Dekompensation (Straffälligkeit, Führerscheinverlust) u.a. mehr. Eine Konsumpause ist auch für die Dauer des rehabilitativen Prozesses unverzichtbar, da in einer konsumfreien Umgebung eine Haltungs- und Einstellungsveränderung in Richtung Abstinenz leichter erzielt werden kann.

Trinkpausen werden von Abhängigen häufig in systematischer Form eingehalten, z. B. zu bestimmten Jahreszeiten (ggf. aus religiösen Motiven) oder zu festen Tageszeiten und Wochentagen (z.B. nicht vor $17 \mathrm{Uhr}$, nicht in der Arbeitszeit, nur am Wochenende). Sie dienen oft der subjektiven Vergewisserung, den Konsum kontrollieren zu können und sind meist ein Indiz für die bereits eingetretene Abhängigkeit.

Weitere Reha-Ziele. Neben diesen basalen Rehabilitationszielen werden weitere Ziele pragmatisch nach der Systematik des biopsychosozialen Modells erarbeitet, z. B. Verbesserung der gesundheitlichen Verfassung, Selbstwertstärkung, Fortschritte in der Beziehungsgestaltung und im Sozialverhalten (Abb.3).

Aus volkswirtschaftlicher Sicht ist die Minderung des postrehabilitativen Akutbehandlungsbedarfs (Vermeidung von Arbeitsunfähigkeitszeiten und erneuten Krankenhausbehandlungen) ein bedeutsames Ziel.
Aus der patientenzentrierten Perspektive haben nicht zuletzt „weiche Faktoren“ wie die Lebenszufriedenheit nach der Behandlung einen hohen Stellenwert (,zufriedene Abstinenz“).

Abstinenz ist das primäre Ziel der medizinischen

Rehabilitation Abhängigkeitskranker.

\section{Behandlungsdauer und Setting}

Die DRV bewilligt und bezahlt stationäre Langzeittherapien mit einer je nach Einrichtungskonzept variablen Behandlungsdauer von 10-16 Wochen, Kurzzeittherapien mit 8 Wochen, ganztägig ambulante Rehabilitation über 12 Wochen, niederfrequente ambulante Rehabilitation bis zu 18 Monaten mit maximal 120 Einzel- und Gruppensitzungen sowie 12 Angehörigengesprächen $[2,5]$.

Nach den Reha-Therapiestandards sollte sich die Dauer und Intensität der Behandlung am Schweregrad orientieren $[6,11,12]$. Katamnesestudien zeigen eine hohe Korrelation des Behandlungserfolgs mit der Dauer der Rehabilitation. Die Abstinenzquote liegt bei einer Behandlungsdauer von $<12$ Wochen bei $34 \%$, bei einer Dauer von $12-16$ Wochen bei $43 \%$ und bei einer Dauer $>16$ Wochen bei 45\% (nach Standard DGSS 4) [23].

Der Erfolg der EBW ist umso erfolgreicher, je länger die Reha-Maßnahmen dauern.

\section{Interventionen}

Das psychopathologische Syndrom „Abhängigkeit“ wird nach klinischem Konsens sowohl in der Phase der Akut- als auch der Entwöhnungsbehandlung mit einer Kombination aus verschiedenen Interventionen durch ein multiprofessionelles Team behandelt (Komplexbehandlung) [8]. Die Einzelinterventionen erfolgen störungsspezifisch bezogen auf die nach biologischen, psychologischen und sozialen Gesichtspunkten klassifizierbaren Störungsbilder (Abb.3), denen wiederum spezifische Verursachungsfaktoren zugrunde liegen, für deren Bewältigung multidisziplinäre Fachkompetenz erforderlich ist $[5,10,13]$.

Wichtig ist die interdisziplinäre Zusammenarbeit verschiedener Fachbereiche.

Integrierte und kombinierte Behandlungen sind wirksamer als Einzelmethoden, wobei zwischen den ver- 


\section{Tipp für die Praxis}

Behandlungsmethoden, die Klienten aktiv in den Behandlungsprozess einbeziehen, sind erfolgreicher. Dabei sind Techniken und Hilfen, die zu einer Stärkung der Ich-Fähigkeiten der Patienten beitragen, zu präferieren [6].

schiedenen Kombinationsformen keine deutlichen Unterschiede bestehen [14].

Therapeutische Haltung (empathisch, nicht bewertend), Beziehungsgestaltung und Milieu sind weitere bedeutsame Wirkfaktoren einer Intervention.

Wirksame psychotherapeutische und psychosoziale Einzel- und Gruppeninterventionsformen sind aus Tab. 3 zu entnehmen. Diese gelten auch für Patienten mit komorbiden psychischen Störungen.

\section{Psychotherapeutische Interventionen}

Motivationale Interventionsformen. Diese sind eine basale psychotherapeutische Herangehensweise an den abhängigen Menschen [15]. Motivationale Interventionsformen (MI) sind eine personenzentrierte (ausgehend von der Sichtweise des Patienten), direktive (d.h. zielstrebige) jedoch nonkonfrontative (d.h. empathische) Methode zur Verbesserung der intrinsischen oder Eigenmotivation für eine Veränderung. Dadurch kann die anfängliche Ambivalenz gegenüber dem therapeutischen Prozess meist überwunden werden. MI vermeiden Widerstand, lösen Ambivalenz auf und leiten Veränderungen ein. Wirkfaktoren sind Problemaktualisierung, Ressourcenaktivierung und aktive Hilfe zur Problembewältigung.

\section{MI ist die psychotherapeutische Domäne der Akutbehandlung.}

In der EWB kommen Elemente einer Motivationsförderung in 14,8\% zur Anwendung [16].

Kognitive Verhaltenstherapie. Die kognitive Verhaltenstherapie (KVT) ist in der EWB mit 85\% die am häufigsten angewandte psychotherapeutische Methode [16]. Nach Süß ist eine verhaltenstherapeutische Breitbandtherapie einer Minimaltherapie oder eklektischen Therapie bzgl. Abstinenz- und Besserungsraten deutlich überlegen [12]. Die Erfolgsquote liegt in Deutschland bei $60 \%$ (international $51 \%$ ), während sie für eklektische Therapie bei $48 \%$ (international $43 \%$ ),

\section{Tabelle 3}

Evidenz für Einzelinterventionen.

\begin{tabular}{|c|c|c|}
\hline Interventionskomponenten & $\begin{array}{l}\text { Empfehlungs- } \\
\text { grad }\end{array}$ & $\begin{array}{l}\text { Level of Evidence } \\
\text { (LoE) }\end{array}$ \\
\hline motivationale Interventionsformen & A & 1a \\
\hline kognitive Verhaltenstherapie & A & 1a \\
\hline Verhaltenstherapie & A & $1 \mathrm{~b}$ \\
\hline psychodynamische Kurzzeittherapie & B & $1 b$ \\
\hline Kontingenzmanagement & B & $2 b$ \\
\hline Paartherapie & A & $1 b$ \\
\hline neurokognitives Training (NKT) & KKP & \\
\hline Angehörigenarbeit & A & $1 b$ \\
\hline angeleitete Patientengruppen & B & $2 b$ \\
\hline
\end{tabular}

für Disulfiram bei 29\% und für Minimaltherapie bei 25\% (jeweils DGSS 4, Kap. 8.1) liegt [7].

Kontingenzmanagement. Dieses Verfahren ist wirksam bzgl. der Aufrechterhaltung der Abstinenz oder einer Trinkmengenreduktion. Bei dieser Vorgehensweise übt der Rehabilitand im Rahmen von Verhaltensverträgen die Ablehnung alkoholassoziierter Reize (z.B. Werbebilder) und die Verstärkung neutraler Stimuli (z.B. Freizeitaktivitäten) [7].

Psychodynamische Kurzzeittherapie. Auch die psychodynamische Kurzzeittherapie hat statistisch signifikante Ergebnisse bzgl. Rückfallprävention, wenn auch mit geringerer Effektstärke [6, 7].

Weitere Verfahren. In Abhängigkeit von speziellen Kliniksettings und dem Ausbildungsstand der Therapeuten kommen weitere Verfahren zur Anwendung. Deren Wirksamkeit ist meist nur bedingt evaluiert. Dazu gehören z.B.

- neurokognitives Training

- Cue-Exposure

- achtsamkeitsbasierte Therapie

- Biofeedback

- systemische Therapieelemente

- Yoga und

- Akupunktur $[6,7]$. 
Da diese Verfahren in der Regel in den therapeutischen Gesamtkontext gut eingepasst sind, werden sie von den Rehabilitanden positiv konnotiert.

\section{Sozialtherapeutische Interventionen}

Stabile soziale Beziehungen und Netzwerke wirken sich positiv auf den Behandlungserfolg aus. Gefährdende Faktoren sind:

- sozialer Stress

- Alkoholbelastung in der Familie

- Partnerschaftsprobleme

- arbeitsplatzbezogene Komplikationen, insbesondere Langzeitarbeitslosigkeit (s. Kap. „Besondere Zielgruppen“)

\section{Tipp für die Praxis}

Flankierende Maßnahmen zur beruflichen und sozialen Stabilisierung bzw. Wiedereingliederung sind auch in der Phase der EWB geboten, da eine Erwerbstätigkeit ein wichtiger Faktor für den Behandlungserfolg ist [5-7].

Einbeziehen der Angehörigen. Für die Klärung innerfamiliärer Dysfunktionalitäten bzw. zur Stabilisierung der sozialen Beziehungen hat sich die Einbeziehung von Angehörigen bewährt. Dazu gehören bspw. Beratung in Angehörigen-, Partner- und Kinderseminaren/ gruppen sowie das Expositionstraining mit therapeutischen Aufgabenstellungen z. B. bei Familienheimfahrten $[11,16]$. Die Interventionen können sogar aufsuchende Sozialarbeit im Wohn- und Arbeitsumfeld unter Einbeziehung von Betriebsangehörigen bzw. Vorgesetzten umfassen.

Beratung und Paartherapie, insbesondere mit verhaltenstherapeutischen Elementen hat sich als zielführend erwiesen $[6,11,16]$.

Therapeutisches Milieu. Der ärztlich-psychologisch verantwortete somato- und psychotherapeutische Prozess sollte deshalb in einem sozialtherapeutisch strukturierten Rahmen („therapeutisches Milieu“) stattfinden. Wirksam sind in diesem Rahmen vornehmlich die therapeutische Beziehung und die Milieutherapie. Von den Einzelinterventionen sind wirksam

- die Psychoedukation für Betroffene und Angehörige,

- der Peer-to-Peer-Ansatz und Trialog,

- das Training von Alltags- und sozialen Fertigkeiten,

- künstlerische Therapien,
- Ergotherapie,

- Sport- und Bewegungstherapie sowie

- das Einüben von Selbsthilfe und verwandter Konzepte $[5,13]$.

Das Arbeiten in geleiteten Patientengruppen, Bibliotherapie und therapeutische Hausaufgaben kommen als ergänzende Maßnahmen infrage $[6,11,16]$. Zu diesem Aufgabenbereich gehört auch die Vorbereitung auf und eine differenzierte Überleitung in die nahtlose Nachsorge oder Adaption oder andere Formen der Postakutbehandlung.

\section{Interventionen zur Rückfallprophylaxe}

Um das übergeordnete Ziel der EWB „Abstinenz“ nicht nur zu erreichen, sondern Gefährdungen im postrehabilitativen Verlauf wirksam begegnen zu können, kommt der psychosozialen Rückfallprophylaxe höchste Bedeutung zu. Die kritische Zuspitzung des Leidensdrucks im Rückfall ist als therapeutische Chance zu nutzen. Insbesondere die positive Bewältigung der „Kränkung“ durch die Verletzung des Abstinenzvorsatzes ist von großer prognostischer Bedeutung. Behandlungsmodule zur Rückfallprophylaxe greifen auf das gesamte Spektrum psycho-, sozial- und pharmakotherapeutischer Interventionsmöglichkeiten zurück (Abb.3).

Für Patienten ohne Abstinenzziel sind dagegen Maßnahmen im Sinne eines „Trinkbeginn-Managements“ zu treffen und ein Notfallplan für den Fall des Scheiterns einer Trinkmengenreduktion zu vereinbaren.

Hilfreich sind im Rahmen der Nachsorge bzw. weiterer Postakutbehandlung:

- eine enge Anbindung an das regionale Suchthilfesystem

- der Anschluss an Selbsthilfegruppen

- die Nutzung von „Hotlines“

- im geeigneten Falle die Einleitung einer Pharmakoprophylaxe (Tab.4).

Bei der medizinischen Rehabilitation Alkoholabhängiger handelt es sich um ein komplexes Interventionspaket mit vorrangig psycho- und sozialtherapeutischen Behandlungsverfahren. 


\section{Besondere Zielgruppen}

Psychische Komorbidität. Begleit- und Folgekrankheiten sind häufig (Tab. 1). Generell ist der klinische Verlauf bei Patienten mit einer psychischen Komorbidität schwerer, die Compliance ist geringer, die Frequenz von Rückfällen und stationären Wiederholungsbehandlungen ist höher, die Therapie-Response ist geringer und die langfristige Prognose ist weniger günstig.

Komorbide psychische Störungen beeinflussen sich gegenseitig negativ, was bei der Behandlungsplanung zu beachten ist $[7,18]$.

Eine eindeutige Empfehlung für ein eher parallel/ gleichzeitiges oder zeitversetzt/konsekutives Setting bei komorbid Erkrankten gibt es nicht [7]. Es spricht jedoch mehr für die Wirksamkeit einer gleichzeitigen Mitbehandlung von komorbiden Störungen, wodurch die Behandlung komplexer wird, was wiederum spezifische Therapiekonzepte voraussetzt.

Bei Komorbidität mit Psychosen wird im kurativen Bereich (Akutbehandlung) eher eine integrierte Behandlung durch ein einziges Team und in einem Setting empfohlen, wobei Pharmakotherapie und Psychoedukation, verbunden mit Elementen der MI gute langfristige Ergebnisse erzielen [18]. Das gilt grundsätzlich auch für die Behandlung von Depressionen, bipolaren Störungen, ADHS, Angststörungen und PTBS. Die Übertragbarkeit dieser Ergebnisse auf die rehabilitativ ausgerichtete EWB ist andererseits begrenzt.

Höheres Alter. Ältere Rehabilitanden zeigen eine höhere Behandlungsadhärenz und damit eine bessere Prognose. Sie leiden mit höherer Wahrscheinlichkeit an somatischer und psychischer Komorbidität [7, 10]. Psychotherapeutisch stehen bei ihnen die typischen Lebensbedingungen wie Vereinsamung, Verlust des beruflichen Status, der körperlichen Unversehrtheit und eine veränderte Beziehungsgestaltung im Fokus (Verlustsituationen). Die Behandlung in der eigenen Altersgruppe ist prognostisch günstiger [7]. Auch wenn zusammenfassend für die EWB dieser Zielgruppe keine Evidenz vorliegt, haben sich doch einige Kliniken erfolgreich mit eigenen Behandlungskonzepten an diese Patientengruppe gewandt.

Spezielle Behandlungskonzepte einiger Kliniken gehen auf die Rehabilitation im höheren Alter ein.

\section{Tabelle 4}

Pharmakotherapie im Rahmen eines Gesamtbehandlungsplans.

\begin{tabular}{|llll|}
\hline $\begin{array}{l}\text { Interventions- } \\
\text { komponenten }\end{array}$ & $\begin{array}{l}\text { Empfehlungs- } \\
\text { grad }\end{array}$ & LoE & Ziel \\
\hline Acamprosat & A & $1 \mathrm{a}$ & $\begin{array}{l}\text { Anticraving, Rückfall- } \\
\text { prophylaxe }\end{array}$ \\
\hline Naltrexon & A & $1 \mathrm{a}$ & $\begin{array}{l}\text { Anticraving, Rückfall- } \\
\text { prophylaxe }\end{array}$ \\
\hline Disulfiram & 0 & $1 \mathrm{~b}$ & $\begin{array}{l}\text { Aversionstherapie } \\
\text { (off Label) }\end{array}$ \\
\hline Nalmefene & KKP & & Trinkmengenreduktion \\
\hline
\end{tabular}

Arbeitslosigkeit. Längere Arbeitslosigkeit ist ein häufig komplizierender Faktor. Zur Verbesserung und Stabilisierung der Fähigkeiten im Berufs- und Arbeitsleben ist eine gezielte Förderung und Begleitung zur Wiederaufnahme einer Arbeitstätigkeit geboten. Die Weiterbehandlung ist für diese Patientengruppe besonders sorgfältig zu planen, da Rückfälligkeit in hohem Maße mit einer gescheiterten beruflichen Wiedereingliederung korreliert. Differenziert werden je nach Arbeitsplatzstatus sozialtherapeutische Gruppeninterventionen zur beruflichen Wiedereingliederung, bei Erwerbslosen die Vorbereitung des beruflichen Alltags durch Belastungs- und Arbeitserprobung sowie Praktika [5].

\section{Tipp für die Praxis}

Abhängige mit Migrationshintergrund profitieren besonders, wenn der soziokulturelle Hintergrund von Rehabilitand und Therapeut übereinstimmt bzw. der Migrationshintergrund bei der Gestaltung von Therapiekonzepten berücksichtigt wird [7]. Eine Behandlung in der Muttersprache kann indiziert sein.

Geschlechtsunterschiede. Frauen profitieren ähnlich wie Männer von Entwöhnungsbehandlungen. Allerdings ist es zweckmäßig, ggf. die Rehabilitation getrennt durchzuführen. So können bei bestimmten Befundkonstellationen Spezialeinrichtungen für Frauen oder Männer vorgeschlagen werden. Mindestanforderung ist eine genderspezifische indikative Gruppentherapie.

Komorbidität ist eine häufige Befundlage. 


\section{Adaption und Nachsorge}

Das Rehabilitationsziel des Rentenversicherungsträgers ist erst dann erreicht, wenn der Versicherte ohne Betreuung und ohne erhebliche Rückfallgefährdung in der Lage ist, in das Alltags- und Erwerbsleben zurückzukehren.

Der nahtlose Übergang in nachsorgende Maßnahmen ist zur Sicherung des erreichten Behandlungserfolgs von großer Bedeutung, da mehr als 80\% der Rückfälle bis zum 6. Monat nach einer Postakutbehandlung stattfinden.

Der 1. Monat nach Entlassung ist mit mehr als 30\% der Rückfälle besonders kritisch [25].

Die Rückfallhäufigkeit reduziert sich kontinuierlich, sodass nahtlos aufeinander abgestimmte Nachsorgeangebote mindestens ein Jahr lang vorgehalten werden sollen.

Adaptionsbehandlung. Eine Adaptionsbehandlung (2. Form der medizinischen Rehabilitation) ist dann indiziert, wenn während der EWB deutlich wird, dass das Rehabilitationsziel aufgrund der spezifischen Auswirkungen und Folgen der Abhängigkeit nicht erreicht wird, z.B. bei erheblicher Rückfallgefährdung durch Wohnungs- und/oder Arbeitslosigkeit. Dann muss im Anschluss daran unter Alltagsbedingungen - institutionell geschützt - erprobt werden, ob der Rehabilitand den Anforderungen des Erwerbslebens und der eigenverantwortlichen Lebensführung gewachsen ist. Eine Adaptionsbehandlung kann auch dann indiziert sein, wenn ein Wechsel des sozialen Umfeldes erforderlich ist (Abb. 4).

Ambulante Nachsorge. Im Unterschied dazu kommt eine ambulante Nachsorge in Betracht, wenn das soziale und berufliche Integrationsziel zwar erreicht ist, jedoch für die Aufrechterhaltung der Abstinenz der Anschluss an eine Selbsthilfegruppe nicht ausreichend erscheint und eine ambulante Psychotherapie nicht indiziert oder nicht ausreichend ist. Es handelt sich bei der Nachsorge um definierte Gruppen- und Einzelgespräche, die der Sicherung und Festigung der in der medizinischen Rehabilitation (EWB oder Adaption) erworbenen Verhaltensweisen und Einstellungen dienen. Dabei treten die therapeutischen Elemente der Rehabilitation zugunsten der Förderung sozialer Kontakte und Aktivitäten der Abhängigkeitskranken zurück [2, 7].

\section{Tipp für die Praxis}

Nachsorge ist nach Konsens insbesondere dann wirksam, wenn die Leistungsangebote gemeindeorientiert vernetzt sind und als Systeminterventionen erfolgen [13].

Eine besondere Form der Vernetzung unterschiedlicher Angebote ist das Modell "Community Reinforcement Approach“ (CRA), das verschiedene verhaltensund familientherapeutische Interventionen in die Suchtbehandlung einbezieht [19].

\section{Wirksamkeit der EWB}

\section{Ergebnismaße}

Sozialmedizinisch. In enger Beziehung zur Therapiezielplanung stehen die Ergebnismaße. Das sozialmedizinisch bedeutsamste Ergebnismaß ist die Beitragszahlung bzw. der Verbleib im Erwerbsleben 2 Jahre nach Abschluss der Behandlung [21]. Diese Erfolgsquote korreliert mit Abstinenz. Die längerfristige postrehabilitative Abstinenz wird nach den Standards des Deutschen Kerndatensatzes im Rahmen von Katamneseerhebungen ermittelt [20].

Katamnestisch. Die katamnestische Erfolgsquote umfasst den Anteil der Patienten, der ein Jahr nach Ende der Behandlung durchgängig abstinent oder abstinent nach kurzem Rückfall ist. Dazu gibt es nach den Standards der Deutschen Gesellschaft für Suchtforschung und Suchttherapie (DGSS) mehrere Berechnungsmethoden. Bei der Variante „DGSS 4“ werden ausschließlich die Rehabilitanden gewertet, über die valide Auskünfte zu den Katamnesezeitpunkten zu erhalten sind, bezogen auf alle Patienten, die zur Behandlung angetreten sind (Intention to treat: ITTAnalyse).

Volkswirtschaftlich. Von volkswirtschaftlichem Interesse ist ferner der weitere Anfall von Arbeitsunfähigkeitszeiten und Krankenhausbehandlungen nach einer Rehabilitation sowie die Kosten-Nutzen-Relation der monetären Aufwendungen insgesamt (Social Return on Investment) [22].

Weitere Ergebnismaße. Hierzu zählen Morbidität, Komorbidität, Mortalität, andere Teilhabekriterien, aber auch Faktoren wie Lebensqualität und Lebenszufriedenheit. Weitere konsumbezogene Ergebnismaße sind Konsumreduktion (Trinktage und -menge), Anteil 
der konsumfreien Tage, Zeit bis zum ersten Rückfall, Anteil der schweren Trinktage u.a. [7, 10].

\section{Ergebnisse von Katamnesen}

Behandlungsaufwendungen und krankheitsbedingte Gesamtkosten ergeben eine positive Kosten-NutzenRelation. Die katamnestischen Erfolgsquoten nach DGSS 4 liegen für die stationäre Reha bei $40 \%$, für die ambulante Reha bei $52 \%$ und die ganztägig-ambulante Reha bei 45\% [22 - 25]. Patienten im ambulanten bzw. ganztags ambulanten Setting verfügen i.d.R. über eine bessere soziale und berufliche Integration, was sich positiv auf die Erfolgsquote auswirkt [24, 25].

Eine Behandlung ist grundsätzlich effektiver als Nichtbehandlung [12, 26].

Die Behandlung der Patienten hat ferner eine verbesserte Gesundheit und Lebensqualität sowie Konsolidierung der Finanzen bei Angehörigen zur Folge [9, 21]. Beitragszahlungen werden nach 2 Jahren von $90 \%$ der Rehabilitanden geleistet [21]. Arbeitsunfähigkeits- und Krankenhaustage gehen im langfristigen Vergleich um bis zu $80 \%$ zurück [7].

Die Erfolgsrate der stationären bzw. ganztags ambulanten EWB ist bei vorangegangener Entzugsbehandlung günstiger [22, 25, 27]. Das Komplikationsrisiko ist nach mehreren Entzugsbehandlungen im Vorfeld erhöht [10]. Rehabilitanden mit diagnostizierter psychischer Komorbidität haben eine schlechtere Erfolgsquote. Wiederholte EWB haben ebenfalls eine schlechtere Prognose, was auf den einsetzenden Chronifizierungsprozess zurückzuführen ist. Trotzdem sollte motivierten Patienten, unter Beachtung der Indikationsregeln, eine erneute Behandlung angeboten werden, da sie nicht grundsätzlich unwirksam ist.

Mit zunehmendem Alter steigt die Erfolgsquote der EWB. Sie beträgt $29 \%$ bei Rehabilitanden bis zum Alter von 40 Jahren und $44 \%$ im Alter von über 40 Jahren [22].

Erwerbstätigkeit unterstützt die Abstinenz und somit den Behandlungserfolg. Erwerbstätige sind nach Rehabilitation im stationären/ganztags ambulanten Setting in 49 bzw. 51 \% abstinent, während Erwerbslose dieses Ziel nur in 31 bzw. 35\% (DGSS 4) erreichen [22, 25].
Die Entwöhnungsbehandlung ist selbst bei konservativer Berechnung eine der erfolgreichsten medizinischen Rehabilitationen.

\section{Andere Formen der Postakut- behandlung}

Neben den medizinischen Rehabilitationsformen (EWB, Adaption), die überwiegend im Auftrag der DRV durchgeführt werden, kommen auch andere Formen der Postakutbehandlung mit anderer Trägerschaft und Finanzierung zum Tragen (s. Box). Diese sollen als Rehabilitationskette eng kooperieren (Abb.4) [2].

\section{Weitere Formen der Postakutbehandlung}

Beratung und Betreuung (SGB divers)

- niederschwellige Hilfen (Grundversorgung, Tagesstruktur etc.)

- Sucht- und Drogenberatung (inkl. Schuldnerberatung)

- sozialpsychiatrische Betreuung (öffentlicher Gesundheitsdienst (ÖGD) oder Wohlfahrt)

- Hilfen zur Erziehung (SGB VIII)

- Suchtberatung im Betrieb

Beschäftigung, Qualifizierung, Arbeitsförderung (SGB III)

- Beschäftigung (u.a. Arbeitsprojekte)

- Qualifizierung

- Arbeitsförderung (Maßnahmen Arbeitsagentur/ Jobcenter)

- berufliche Rehabilitation

- Beschäftigung für behinderte Menschen (in Werkstatt)

Eingliederungshilfe (SGB XII)

- ambulant betreutes Wohnen

- stationäres sozialtherapeutisches Wohnen

- Übergangswohnen (zeitlich befristet)

- tagesstrukturierende Maßnahmen

\section{Ambulante Behandlungsformen}

Ambulante Behandlungen finden sowohl vor als auch nach einer Rehabilitation in der hausärztlichen Praxis, einer psychiatrischen Institutsambulanz oder einer psychosozialen Beratungsstelle statt. Eine verbindliche Überleitung ist in beide Richtungen nahtlos anzustreben $[1,2,6]$. 


\section{Zielgruppen}

Einen besonderen Hilfebedarf haben folgende

Alkoholabhängige:

- mit somatischen und psychischen komorbiden Störungen

- mit (komorbider) Glücksspiel- und Medienproblematik

- mit Vermittlungshemmnissen in der Arbeitswelt

- in besonderen sozialen Lebenslagen, insbesondere ohne Wohnung

- im Alter über 50 Jahre mit Missbrauch von Medikamenten und somatischen Störungen

- in Haft

- die chronisch mehrfach beeinträchtigt sind (CMA)

- mit Kindern und in Schwangerschaft

- im Jugend- und jungen Erwachsenenalter insbesondere bei gleichzeitigem Missbrauch von illegalen Drogen [9]

Beratung wird in Kostenträgerschaft von Kommunen und Ländern in Schul- und Erziehungsberatungsstellen sowie bei Jugendämtern, Fahreignungs- und Justizbehörden angeboten (Abb. 1) [13].

Bei psychischer Komorbidität ist ggf. eine Richtlinienpsychotherapie indiziert. Beispielsweise integriert die alkoholismusspezifische Psychotherapie Methoden des MI, der KVT, des Community Reinforcement Approach und der Selbsthilfe [28]. In besonderen Fällen schließt sich eine weitere ambulante oder stationäre Rehabilitation an (Auffrischungstherapie).

Zusätzliche Medikation. Von Bedeutung ist auch die pharmakagestützte Rückfallprophylaxe (Tab.4). Eine Trinkmengenreduktion (Anzahl schwerer Trinktage sowie konsumierte Menge pro Trinktag) ist für Nalmefene bei Sicherstellung einer regelmäßigen psychosozialen Beratung belegt. Acamprosat erwies sich insbesondere bei psychisch unauffälligen, funktional konsumierenden Abhängigen als wirksam. Naltrexon hat statistisch signifikante, jedoch eher kleine Effekte bezüglich des Zeitraums bis zum ersten Rückfall sowie der Anzahl schwerer Trinktage. Bezüglich der Rückfallraten nach 6 Monaten konnte eine moderate Überlegenheit der Kombination von Naltrexon mit Acamprosat festgestellt werden [7].

Eine Verschreibung von Disulfiram ist „off-label“ mit den entsprechenden Indikationseinschränkungen und Aufklärungsmaßnahmen als Heilversuch bei fehlenden Alternativen möglich.

\section{Tipp für die Praxis}

Eine medikamentöse Behandlung sollte grundsätzlich von anderen psychosozialen Interventionen begleitet werden [10].

Gerade schwer abhängige Patienten wünschen nicht selten eine medikamentöse Verordnung im Sinne einer Ultima Ratio.

Auch wenn statistische Signifikanz für die Wirkung pharmakologischer Interventionen vorliegt, ist die klinische Signifikanz bisher nicht überzeugend, was sich in der zögerlichen Verordnung der Substanzen niederschlägt.

\section{Sozialrehabilitative Postakutbehandlung}

Trotz (wiederholter) EWB kann der Krankheitsverlauf chronifizieren, denn nur dauerhafte Abstinenz beendigt den Schädigungsprozess (Abb.4). Da dieses Ziel nicht von allen Abhängigen und wenn, oft nur punktuell oder auf einzelne Lebensphasen begrenzt, erreicht wird, gilt es, mit flankierenden sozialtherapeutischen Maßnahmen das weitere Fortschreiten des körperlichen und psychosozialen Verfalls zu begrenzen.

Formen dieser speziellen Rehabilitation sind Systeminterventionen wie multiprofessionelle gemeindepsychiatrische Versorgungsansätze, Case-Management, Arbeitsrehabilitation und Maßnahmen zur Teilhabe am Arbeitsleben, Bildungsangebote zum Nachholen von Schulabschlüssen, beschützende Wohnformen einschließlich stationäre sozialtherapeutische Einrichtungen sowie Angebote der Eingliederungshilfe und niedrigschwellige Hilfeangebote (z. B. Überlebenshilfen, Tagesstätten, Freizeitangebote) [13].

\section{Tipp für die Praxis}

Insbesondere für CMA können Übergangseinrichtungen (soziotherapeutische Einrichtungen) in Kostenträgerschaft der überörtlichen Träger der Sozialhilfe indiziert sein [7, 13].

\section{Berufliche Rehabilitation}

Ein wichtiges Versorgungssegment ist die Förderung der Teilhabe am Arbeitsleben für arbeitslose Alkoholkranke u.a. durch Grundsicherungsstellen, Jobcenter und die Agentur für Arbeit. Bei entsprechender Indika- 
tion kommen auch berufliche Rehabilitationsmaßnahmen für Alkoholabhängige infrage, die einer gesonderten Antragstellung bedürfen (Abb.4).

Berufsfördernde Nachsorgeleistungen, wie z. B. stufenweise Wiedereingliederung am letzten Arbeitsplatz oder Antrag auf Leistungen zur Teilhabe am Arbeitsleben, sind im Einzelfall zu prüfen und ggf. einzuleiten $[5,6]$.

\section{Selbsthilfe(gruppe)}

Der Besuch von Selbsthilfegruppen setzt in der Regel einen Abstinenzwillen voraus (Abb.4). Er unterstützt professionelle Hilfen und verbessert den langfristigen Behandlungserfolg $[7,13]$. Zugangshindernisse müssen aktiv angesprochen und noch während der stationären Behandlung geklärt werden. Ziel der Selbsthilfe ist, die psychische Widerstandsfähigkeit (Resilienz) durch die Erfahrung der eigenen Stärke (Empowerment) zu mobilisieren und für die Aufrechterhaltung der Abstinenz zu nutzen. Es können auch andere Formen der Selbsthilfe unter Nutzung moderner Medien infrage kommen $[2,7,9]$.

Selbsthilfe fördert die soziale Integration, überwindet Isolation, verbessert Kommunikation und vermittelt Wissen und Kompetenzen.

\section{Kernaussagen}

Die Entwöhnungsbehandlung ist eine medizinische Rehabilitationsmaßnahme. Die Notwendigkeit muss ärztlich begründet, die Durchführung vom zuständigen Kostenträger bewilligt werden. Versicherungsrechtliche Voraussetzungen (z. B. Beitragszahlungen im Vorfeld) und gesundheitliche Eignung (z. B. Belastbarkeit, Reha-Fähigkeit, günstige Prognose) müssen gegeben sein. Die Behandlung erfolgt als komplexes multiprofessionelles und multimodales Interventionspaket, das alle Dimensionen des biopsychosozialen Krankheitsmodells umfasst. Primäres Ziel der Rehabilitation ist die Reintegration in den regulären Arbeitsmarkt, sodass Beiträge in die Rentenversicherung gezahlt werden können. Eine erfolgreiche Arbeitsplatzintegration korreliert hoch mit einer abstinenten Lebensweise. Wenn das Ziel „Abstinenz“ nicht alleine durch eine Entwöhnungsbehandlung erreicht wird, kann sich eine Adaption oder eine Nachsorge anschließen. Die Entwöhnungsbehandlung ist aus sozialmedizinischer Sicht eine der erfolgreichsten medizinischen Rehabilitationen.

\section{Über den Autor}

\section{Heribert Fleischmann}

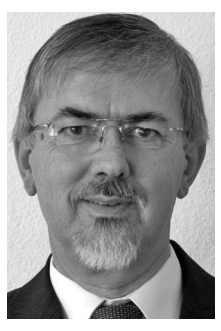

Dr. med. Jahrgang 1951. 1978 Staatsexamen und Promotion, 1984 Nervenarzt, 1986 Zusatzbezeichnung Psychotherapie, 1992 systemische Therapie, 1994 Facharzt für psychosomatische Medizin. Seit 1998 ÄD Bezirksklinikum Wöllershof. Seit 1989 Mitglied der Deutschen Gesellschaft für Suchtforschung und Suchttherapie (DGS), seit 1989 Mitglied des Suchtausschusses der Bundesdirektorenkonferenz, seit 2003 Delegierter der BAG im Vorstand der DHS, seit 2006 Stellvertretender Sprecher des Referates Sucht der DGPPN, seit 2009 Vorsitzender der DHS.

Interessenkonflikt: Der Autor ist seit 1986 Itd. Arzt einer Rehabilitationseinrichtung, die von der DRV belegt wird. Er ist Vorsitzender der DHS und damit Interessenvertreter für Prävention, Behandlung und Rehabilitation Suchtkranker. Er war in die Entwicklung der S3-Leitlinie „Screening, Diagnose und Behandlung alkoholbezogener Störungen" eingebunden. Wirtschaftliche Vorteile bezieht er aus diesen oder anderen Tätigkeiten nicht und hat sie auch zu keinem früheren Zeitpunkt bezogen. Als Ärztlicher Direktor und Chefarzt nimmt er keine Erfolgsbeteiligung in Anspruch.

\section{Korrespondenzadresse}

Dr. med. Heribert Fleischmann

Bezirksklinikum Wöllershof

Wöllershof 1

92721Störnstein

E-Mail: Heribert.Fleischmann@medbo.de

\section{Literatur}

1 Deutsche Rentenversicherung Bund. Rehabilitationsbedürftigkeit und indikationsübergreifende Problembereiche - Leitlinien für den Beratungsärztlichen Dienst der Deutschen Rentenversicherung Bund. www.deutsche-rentenversichung.de (Stand Oktober 2005)

2 Deutsche Rentenversicherung Bund. Rahmenkonzept zur medizinischen Rehabilitation in der gesetzlichen Rentenversicherung. www.deutsche-rentenversichung.de (Stand April 2009) 
3 Friebös RM, Zaudig M, Nosper M, Hrsg. Rehabilitation bei psychischen Störungen. München, Jena: Urban und Fischer; 2005

4 Deutsche Hauptstelle für Suchtfragen e. V. Hrsg. Jahrbuch Sucht 2014. Lengerich: Pabst; 2014

5 Deutsche Rentenversicherung Bund. Leitlinien für die sozialmedizinische Beurteilung bei Abhängigkeitserkrankungen. www.deutsche-rentenversichung.de (Stand April 2010)

6 Deutsche Rentenversicherung Bund. Reha-Therapiestandards Alkoholabhängigkeit - Leitlinie für die medizinische Rehabilitation der Rentenversicherung. www.deutsche-rentenversi chung.de (Stand Januar 2011)

7 DGPPN. S3-Leitlinie „Screening, Diagnose und Behandlung alkoholbezogener Störungen“. AWMF-Register Nr. 076-001 2014

8 Mann K, Grosshans M. Akutbehandlung des Alkoholentzugs. Psychiatrie Psychother up2date 2011; 5: 73-84

9 Deutsche Hauptstelle für Suchtfragen e. V. Suchthilfe und Versorgungssituation in Deutschland. www.dhs.de

10 Alcohol-use disorders: Diagnosis, assessment and management of harmful drinking and alcohol dependence (CG115). London/UK: National Institute for Health and Clinical Excellence (NICE); 2011

11 Bottlender M, Köhler J, Soyka M. Effektivität psychosozialer Behandlungsmethoden zur medizinischen Rehabilitation alkoholabhängiger Patienten. Fortschr Neurol Psychiatrie 2005; 73: $1-13$

12 Süß HM. Zur Wirksamkeit der Therapie bei Alkoholabhängigen: Ergebnisse einer Meta-Analyse. Psychologische Rundschau 1995; 46: 248-266

13 DGPPN, Hrsg. S3-Leitlinie Psychosoziale Therapie bei schweren psychischen Erkrankungen. AWMF-Register Nr. 038-020 2012

14 Anton RF, O'Malley SS, Ciraulo DA et al. COMBINE Study Research Group. Combined pharmacotherapies and behavioral interventions for alcohol dependence: the COMBINE study: a randomized controlled trial. J Am Med Assoc 2006; 295: 2003-2017

15 Frischknecht U, Kiefer F. Motivationstherapie bei Alkoholkranken. Psychiatrie Psychother up2date 2011; 5: 321-332

16 Schmidt P, Köhler J, Soyka M. Evidence-based treatments in the inpatient rehabilitation of alcoholics. Fortschr Neurologie Psychiatrie 2008; 76: 86-90

17 Gouzoulis-Mayfrank E. Komorbidität von Psychose und Sucht. Psychiatrie Psychother up2date 2010; 4: 81-95

18 Preuss UW. Abhängigkeitserkrankungen und Psychosen: Erkenntnisse und Kontroversen in Vorbereitung auf neue Klassifikationssysteme. Die Psychiatrie 2008; 2: 61-68

19 Lange W, Reker M, Driessen M. Community Reinforcement Approach (CRA) - Überblick über ein integratives Konzept zur
Behandlung von Abhängigkeitserkrankungen. Sucht 2008; 54: $20-30$

20 Deutsche Hauptstelle für Suchtfragen e. V. Hrsg. Deutscher Kerndatensatz zur Dokumentation im Bereich der Suchtkrankenhilfe - Definitionen und Erläuterungen zum Gebrauch. Stand: 05.10.2010. Lengerich: Verlag Pabst Science Publishers; 2013: $88-130$

21 Beckmann U, Naumann B. Deutsche Hauptstelle für Suchtfragen e. V. Hrsg. Suchtrehabilitation durch die Rentenversicherung. Jahrbuch Sucht 2014. Lengerich: Verlag Pabst Science Publishers; 2013: 231-247

22 Missel P, Jung C, Herder F et al. Effektivität der stationären Suchtrehabilitation - FVS-Katamnese des Entlassjahrgangs 2011 von Fachkliniken für Alkohol- und Medikamentenabhängige. Sucht aktuell 2014; 1: 5-18

23 Koch A, Kerschl V, Medenwaldt J. Verbandsauswertung des buss - Basis- und Katamnesedaten zu den Entlassungsjahrgängen 2005-2010. Konturen 2011; 1: 32-43

24 Lange N, Neeb K, Missel P et al. Effektivität der ambulanten Suchtrehabilitation - FVS-Katamnese des Entlassjahrgangs 2011 von Ambulanzen für Alkohol- und Medikamentenabhängige. Sucht aktuell 2014; 1: 25-33

25 Schneider B, Mielke D, Bachmeier R et al. Effektivität der Ganztägig ambulanten Suchtrehabilitation - FVS-Katamnese des Entlassjahrgangs 2011 aus Einrichtungen Alkohol- und Medikamentenabhängiger. Sucht aktuell 2014; 1: 34-43

26 Müller-Fahrnow W, Spyra K, Stoll S et al. Sucht-Rehabilitation in Deutschland: Bestandsaufnahme und Perspektiven. Eine Expertise des Lehrstuhls Versorgungssystemforschung, Humboldt-Universität zu Berlin. Verband Deutscher Rentenversicherungsträger 2002. Bd. 32. Berlin: DRV-Schriften; 2002

27 Mann K, Lemenager T, Hoffmann S et al. The PREDICT Study Team. Results of a double-blind, placebo-controlled pharmacotherapy trial in alcoholism conducted in Germany and comparison with the US COMBINE study. Addiction Biology 2013; 18: $937-946$

28 Brueck R, Mann K, Frick K. Alkoholismusspezifische Psychotherapie. Manual mit Behandlungsmodulen. Köln: Deutscher Ärzte-Verlag; 2006

29 Wienberg G, Andritsch F, Bertram W et al. Abhängigkeitskranke in psychiatrischer Krankenhausbehandlung. Ergebnisse einer Erhebung in 14 Kliniken der Bundesrepublik. Sucht 1993; 39: $264-275$

30 Steppan M, Brand H, Künzel J et al. Jahresbericht der Deutschen Suchthilfestatistik 2012. München: IFT Institut für Therapieforschung; 2013 


\section{CME-Fragen}

\section{CME.thieme.de}

\section{CME-Teilnahme}

- Viel Erfolg bei Ihrer CME-Teilnahme unter http://cme.thieme.de

- Diese Fortbildungseinheit ist 12 Monate online für eine CME-Teilnahme verfügbar.

- Sollten Sie Fragen zur Online-Teilnahme haben, unter http://cme.thieme.de/hilfe finden Sie eine ausführliche Anleitung.

\section{1}

Welche Aussage ist falsch?

2
A Die Entwöhnungsbehandlung ist die häufigste Postakutbehandlung.
B Zuständig ist die Deutsche Rentenversicherung (DRV).
C Die Finanzierung ist im SGB VI geregelt.
D Die Klassifikation der Funktionsstörungen erfolgt mit der ICD-10.
E Es gilt die Vereinbarung Abhängigkeitskranker.
A Die DRV entscheidet nach pflichtgemäßem Ermessen über den Antrag.
B Für die Leistungszuweisung muss ein Antrag gestellt werden.
C Alle Suchtkranken haben den aufwendigen Hilfebedarf einer EWB.
D Mit Alkoholkranken über 65 Jahre ist verstärkt zu rechnen.
E Es sind versicherungsrechtliche Voraussetzungen zu klären.

3

Welche Aussage ist falsch? Die Inanspruchnahme einer EWB setzt beim Antragsteller Folgendes voraus:

4

Welche Aussage ist falsch? Die ICF klassifiziert...
A Rehabilitationsbedürftigkeit (Funktionsstörungen bei Abhängigkeit)
B Rehabilitationsfähigkeit (Bereitschaft/Motivation und Belastbarkeit)
C eine günstige Prognose (das Rehabilitationsziel muss erreichbar sein)
D Maßnahmen der Beratung und Motivierung im Vorfeld
E ein körperliches Entzugssyndrom
A Körperstrukturen.
B Krankheitsbeschwerden.
C Aktivitäten.
D Körperfunktionen.
E Kontextfaktoren.

\section{5}

Welche Aussage ist richtig? Behandlungsziele der EWB sind...
A Besserung der Entzugserscheinungen.
B Erhalt der Arbeitsfähigkeit.
C Abstinenz.
D kontrolliertes Trinken.
E Rehabilitationsfähigkeit.

\section{6}

Welche Aussage ist falsch? Wirksame Einzel- und Gruppeninterventionen sind...
A motivationale Interventionsformen.
B kognitive Verhaltenstherapie.
C internetbasierte Therapie.
D psychodynamische Kurzzeittherapie.
E Angehörigenarbeit. 


\section{CME-Fragen}

Entwöhnungsbehandlung Alkoholabhängiger und andere Formen der Postakutbehandlung

\section{7}

Was zählt zu den sozialtherapeutischen Interventionen? Welche Aussage ist richtig?
A neurokognitives Training
B Behandlung mit Acamprosat
C Trinkmengenreduktion
D Milieutherapie
E Biofeedback

\section{8}

Welches ist das vorrangige Ergebnismaß der EWB? Welche Aussage ist richtig?
A Trinkmengenreduktion
B durchgängige Abstinenz
C Trinktage/Monat
D Beitragszahlung in die Rentenversicherung
E Abstinenz nach einem Rückfall

Welche Aussage ist richtig? Die katamnestischen Erfolgsquoten nach DGSS 4 liegen für...
A die stationäre Reha bei $66 \%$.
B die ambulante Reha bei $58 \%$.
C die ganztägig ambulante Reha bei $35 \%$.
D die stationäre Reha bei $40 \%$.
E die ambulante Reha bei 33\%.

Welche Aussage ist falsch? Zu den Postakutbehandlungen zählen...
A die Entwöhnungsbehandlung.
B die medikamentöse Rückfallprophylaxe.
C Richtlinienpsychotherapie.
D die Entzugsbehandlung.
E Eingliederungshilfen. 